\title{
Research in Depolarization and Extinction Coefficient of Particles in Tibetan Plateau by Lidar
}

\author{
Guangyao DAI, Xiaoquan SONG, Xiaochun ZHAI, Songhua WU* \\ ${ }^{1}$ Ocean University of China, Qingdao 266100, China, *Email: wush@ouc.edu.cn
}

\begin{abstract}
Vertical profiles of the depolarization ratio and the extinction coefficient of atmospheric particles in Tibetan Plateau were measured with the OUC Water Vapor, Cloud and Aerosol Lidar during the $3^{\text {rd }}$ Tibetan Plateau Atmospheric Expedition Experiment Campaign in 2013 and 2014. The cloud types and phases, the spatial temporal distribution of the aerosols and the boundary layer height in the Tibetan Plateau were obtained using polarization lidar technique. In this paper, the depolarization ratio was validated with CALIOP polarization simultaneous data, and the extinction coefficient was retrieved by the Fernald method. The result implied that the atmosphere in the Tibetan Plateau was quite clean with low aerosol load and serious pollution. The ice-water mixed cumulus, water cumulus or stratus clouds in Litang and Nagqu were occurred and classified, respectively. The boundary layer height in Nagqu at average altitude over $4600 \mathrm{~m}$ was obtained at around $200 \mathrm{~m}-300 \mathrm{~m}$, which was commonly lower than that in other observed sites.
\end{abstract}

\section{INTRODUCTION}

Atmospheric aerosol particles have a complicated influence on the earth climate by directly absorbing and scattering the atmospheric radiation and by indirectly serving as cloud condensation nuclei 11 . The Tibetan Plateau lies at a critical and sensitive area, which influences the atmosphere in East Asia area and even the whole northern hemisphere. So it is significant to study the impact of aerosol on atmospheric condition and composition in Tibetan Plateau. The polarization lidar technique ${ }^{[2]}$ has been an excellent method of atmospheric probing, which plays an important role in the detection of spatial and temporal distribution of aerosol and cloud phase. In general, it bases on the particle polarization effect, that is, the polarization orientation of backscatter signal of the spherical particles is the same as the linearly polarized laser pulse while the polarization orientation of the non-spherical particle is changed and consists of perpendicular and parallel signals.
The ratio of these two signals corrected by the calibration factor is referred to as the particle linear depolarization ratio. Liu et al..$^{[3]}$ indicated the feasibility of using backscatter coefficients and backscatter color ratios to distinguish aerosols from clouds, which showed clouds have larger backscatter coefficient $(\geq 0.01)$ and higher color ratio around 1 than mostly aerosol types except for optically thin cloud, and the cumulus or stratus clouds generally have higher backscatter coefficients than cirrus. Sassen et al. ${ }^{[4]}$ stated that different cloud phases correspond to differentiable depolarization ratio range, which can be an essential basis to distinguish cloud phases. With the help of particle linear depolarization ratio and extinction coefficient (or backscatter coefficient), the cloud types and phases, the spatial temporal distribution of the aerosols in observed site can be determined, supplying useful and synergistic data to other kinds of aerosol lidars.

\section{METHODOLOGY}

\subsection{POLARIZATION CHANNEL SETUP DESCRIPTION}

The OUC Water Vapor, Cloud and Aerosol Lidar (WVCAL) is multifunctional, and the detailed introduction can refer to a parallel paper in this conference. Fig. 1 presents the schematic diagram of the polarization channel setup. The laser transmitter is a Nd:YAG laser, which produces a laser pulse at $532 \mathrm{~nm}$ with energy per pulse of $120 \mathrm{~mJ}$ and a repetition rate of $30 \mathrm{~Hz}$.

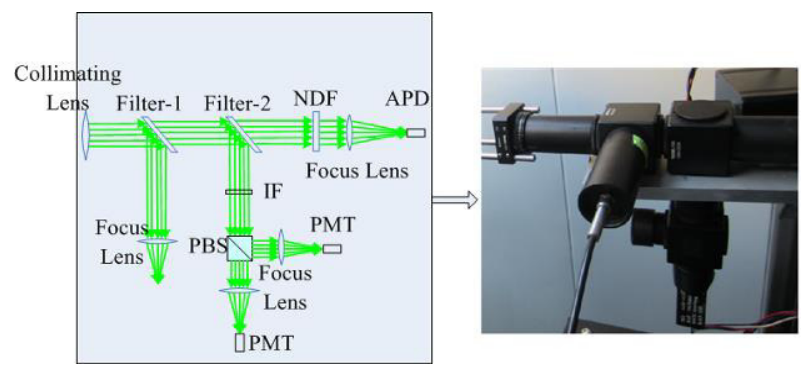

Fig.1. Schematic diagram of the polarization channel of WVCAL system at $532 \mathrm{~nm}$. 
The beam divergence angle is $0.05 \mathrm{mrad}$. The telescope has an aperture of $308 \mathrm{~mm}$ with field-ofview of $1.3 \mathrm{mrad}$. After the backscatter signals are collected by telescope and split by spectrophotometer system, the $532 \mathrm{~nm}$ signal is transmitted to the polarizing beam splitter (PBS) and is separated to $P_{/ /}$and $P_{\perp}$.

\subsection{RETRIEVAL OF DEPOLARIZATION RATIO AND EXTINCTION COEFFICIENT}

The linear volume depolarization ratio $\delta^{v}$ is commonly defined as the ratio of the total perpendicular-polarized backscatter power $\left(P_{\perp}\right)$ to total parallel-polarized backscatter power $\left(P_{/ /}\right)$, measured with polarization lidar by ${ }^{[5]}$ :

$$
\delta^{v}=\frac{\beta_{\perp}}{\beta_{/ /}}=\frac{P_{\perp}}{P_{/ /}}
$$

According to the lidar equation (2):

$$
P=\frac{C \Delta r}{r^{2}}\left(\beta_{a}+\beta_{m}\right) \exp \left\{-2 \int_{0}^{r}\left[\alpha_{m}^{\lambda}(z)+\alpha_{a}^{\lambda}(z)\right] d z\right\}
$$

Where $C$ is the system constant, $\beta$ is the backscatter coefficient, $\alpha$ is the extinction coefficient, the subscripts $a$ and $m$ represent aerosol and molecular, respectively, and $\Delta r$ is the range resolution. The $P_{/ /}$and $P_{\perp}$ can be described as (3) and (4), respectively:

$$
\begin{aligned}
P_{/ /}=\frac{C_{/ /} \Delta r}{r^{2}}\left(\beta_{a}^{\prime \prime}+\beta_{m}^{\prime \prime}\right) & \exp \left\{-2 \int_{0}^{r}\left[\alpha_{m}^{\prime \prime}(z)+\alpha_{a}^{\prime \prime}(z)\right] d z\right\} \\
P_{\perp}=\frac{C_{\perp} \Delta r}{r^{2}}\left(\beta_{a}^{\perp}+\beta_{m}^{\perp}\right) & \exp \left\{-2 \int_{0}^{r}\left[\alpha_{m}^{\perp}(z)+\alpha_{a}^{\perp}(z)\right] d z\right\} \\
& \alpha_{a}(z)+\frac{S_{a}(z)}{S_{m}} \alpha_{m}(z)= \\
& \frac{S_{a}(z) P(z) z^{2} \exp \left\{-2 \int_{z_{0}}^{z}\left[\frac{S_{a}(\zeta)}{S_{m}}-1\right] \alpha_{m}(\zeta) d \zeta\right\}}{\alpha_{a}\left(z_{0}\right)+\frac{S_{a}\left(z_{0}\right)}{S_{m}} \alpha_{m}\left(z_{0}\right)}-2 \int_{z_{0}}^{z} S_{a}(\zeta) P(\zeta) \zeta^{2} \exp \left\{-2 \int_{z_{0}}^{\zeta}\left[\frac{S_{a}(\xi)}{S_{m}}-1\right] \alpha_{m}(\xi) d \xi\right\} d \zeta
\end{aligned}
$$

Where $C_{/ /}$and $C_{\perp}$ are system constants for parallel-polarized channel and perpendicularpolarized channel, respectively.

$C_{/ /}$and $C_{\perp}$ represent the different receiver efficiency on the two channels including the PBS cross-talk effect, and the lidar system depolarization effects including non-linear polarized laser source and optical device depolarization effect. As a result, $\delta^{v}$ cannot be used directly to classify the aerosol types and cloud phases. To retrieve the depolarization properties of particles, the particle linear depolarization ratio $\delta^{p}$ was defined, and the relationship between $\delta^{p}$ and $\delta^{v}$ can be described as (5) :

$$
\delta^{P}=a * \delta^{v}+b
$$

The calibration factors $a$ and $b$, which depend on the system itself, were obtained by WVCAL and CALIPSO validation experiment. ${ }^{[6]}$

Based on the signal of the polarization lidar at $532 \mathrm{~nm}$, the aerosol extinction coefficient can be calculated by the Fernald method [7], and can be written as equation (6):
Where $S_{m}=8 \pi / 3$ and $S_{a}(\mathrm{z})$ are the extinction to backscatter ratios for Rayleigh and Mie scattering, respectively. $S_{a}(\mathrm{z})$ was assumed as a constant and was independent of the range.

The reference height $z_{0}$ was chosen where the atmospheric layer was relative clean with rare aerosols. In this paper, the Minimum Value
Method was used to find out $z_{0}$, that is, $z_{0}$ was the height which minimizes $P(\mathrm{z}) \mathrm{z}^{2} / \beta_{m}(\mathrm{z})$. An iteration was began with $\beta_{a \text {,input }}\left(\mathrm{z}_{0}\right)=4 \times 10^{-10} \mathrm{~km}^{-1} \mathrm{sr}^{-1}$ after the reference height $z_{0}$ had been fixed. The $\beta_{\text {a.input }}\left(\mathrm{z}_{0}\right)$ increased by $10 \%$ for each iteration 
step until the relative difference between $\beta_{a, \text { input }}\left(\mathrm{z}_{0}\right)$ and $\beta_{a, \text { cal }}\left(\mathrm{z}_{0}\right)$ was less than $5 \%{ }^{[8]}$.

The extinction coefficients of the lidar were compared with the CALIOP polarization data. One study case measured on September 30, 2013 was shown in Fig.2. The black dashed line represents the lidar data and the other color lines represent CALIOP polarization data, which shows good consistency.

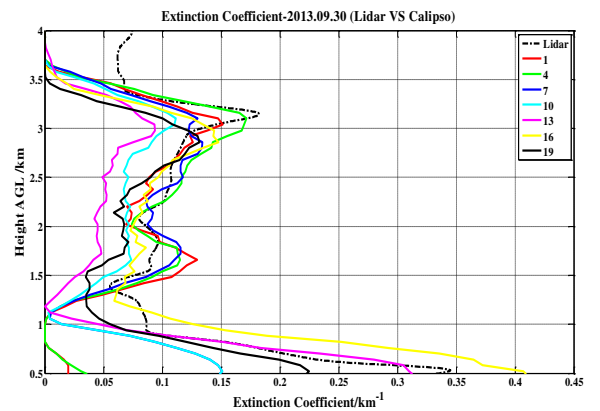

Fig.2. Comparison of extinction coefficient measured by lidar and CALIPSO on Sep 30, 2013

\section{RESULTS}

In this section, some cases including information about particle liner depolarization ratio, extinction coefficient and boundary layer height is discussed.

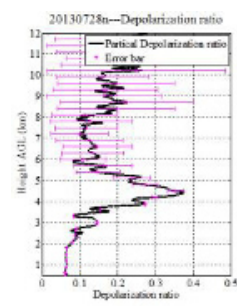

(a)

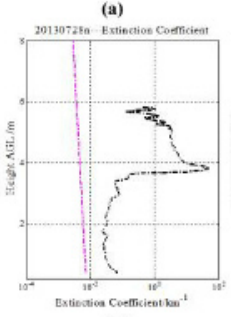

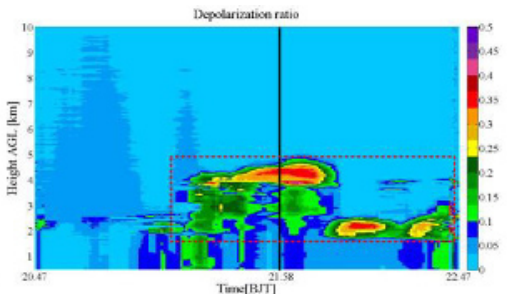

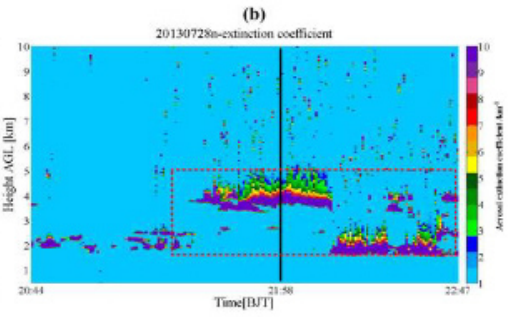

Fig.3.The depolarization ratio and extinction coefficient measured on July 28, 2013 (In Litang). (a) Profile of particle depolarization ratio (black line) and the error bar (pink line). (b) Temporal development of particle depolarization ratio. (c) Profile of $\alpha_{a}$ (black dashed line) and $\alpha_{m}$ ( pink dashed line). (d)

Observation of aerosol extinction coefficient $\alpha_{a}$
In Fig.3, the $\delta^{P}$ at the height of $2-5 \mathrm{~km}$ above ground level (in red dashed box) was in the range of 0.1 to 0.35 . Meanwhile, $\alpha_{a}$ was bigger than 5 $\mathrm{km}^{-1}$. It was implied that clouds existed and can be classified as mixed phase cumulus clouds ${ }^{[9]}$. Furthermore, the $\delta^{P}$ near the ground was less than 0.1 and even less than 0.05 , and $\alpha_{a}$ was about $0.05 \mathrm{~km}^{-1}$. So it was confirmed that the aerosol loading near the ground in Litang was rare, and the aerosols were free of biomass burning aerosols and pure dust.

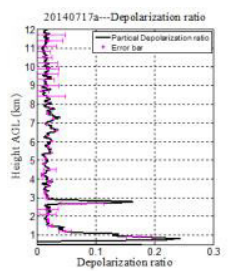

(a)

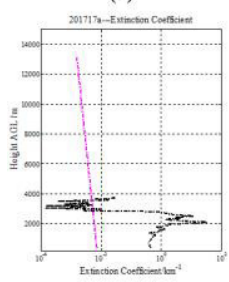

(c)
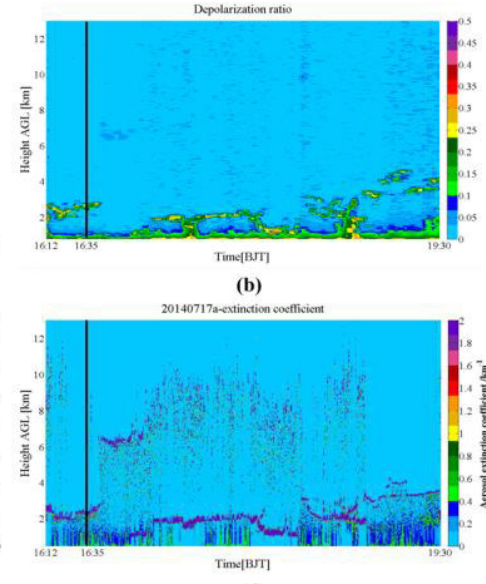

(d)
Fig.4.The depolarization ratio and extinction coefficient measured on July 17, 2014 (In Nagqu) (a)(d) are same as Figure 3 (a)-(d)

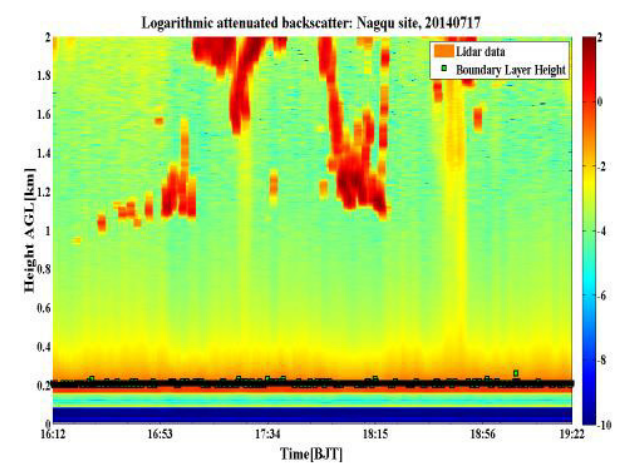

Fig.5. The logarithmic attenuated backscatter and the Boundary Layer Height (BLH) on July 17, 2014 (In Nagqu)

In Fig.4, the $\delta^{P}$ at the height of $2-3 \mathrm{~km}$ above ground level was of 0.05 to 0.15 . Meanwhile $\alpha_{a}$ was bigger than $5 \mathrm{~km}^{-1}$. So it was confirmed that clouds can be classified as water clouds or 
mixed phase clouds dominated by water clouds, and the cloud type can be classified as cumulus or stratus clouds [10]. Except the clouds, the atmosphere above $1 \mathrm{~km}$ was very clean and the depolarization ratios $\delta^{P}$ were close to zero. The air above $1 \mathrm{~km}$ was free or contains tiny of the non-spherical particles. Furthermore, the $\delta^{P}$ near the ground was less than 0.1 and even less than 0.05 and $\alpha_{a}$ was about $0.5 \mathrm{~km}^{-1}$. So it was implied that the air conditions in Nagqu were quite clean and had no other types of aerosols and serious pollution. Fig. 5 showed the boundary layer height in Nagqu at that period was about $200 \mathrm{~m}-300 \mathrm{~m}$, which was commonly lower than that in other observed sites.

\section{CONCLUSIONS}

The key conclusions of the study were listed below:

The extinction coefficients of WVCAL were compared with CALIOP polarization data, which showed good consistency. In the process of clouds classification, $\alpha_{a}$ can generally distinguish aerosols from clouds, and further classify cloud types. $\delta^{p}$ can then judge the cloud phases. In the Tibetan plateau experien, the ice-water mixed cumulus clouds, water cumulus or stratus clouds in Litang and Nagqu were observed and classified. Most of $\delta^{p}$ near ground in Tibetan Plateau were less than 0.1 and even 0.05 , showing that the aerosol loading near the ground was rare and the aerosols were free of biomass burning aerosols and pure dust. Moreover, the atmosphere above 1 $\mathrm{km}$ was very clean and the $\delta^{p}$ was close to zero. The air above $1 \mathrm{~km}$ was free or contains tiny of the non-spherical particles. The boundary layer height in Tibetan Plateau was about 200m-300m, which was commonly lower than that in other observed sites.

\section{ACKNOWLEDGEMENT}

The observation in Tibetan Plateau was completed by the cooperation of CAMS/ LAWS(Chinese Academy of Meteorological Science/Laboratory of Severe Weather) and Ocean University of China (OUC). We thank researcher Liping Liu from CAMS for his help and support. And we thank Meteorological Administrations of Litang and Nagqu for their support.

\section{REFERENCES}

[1] Charlson, R.J., Schwartz, S.E., Hales, J.M., Cess, D., Coakley, J.A., Hansen, J.E., 1992. Climate forcing by anthropogenic aerosols. Science 255, 423-430.

[2] Sassen K., 2005: Polarization in lidar. Springer New York.

[3] Liu, Zhaoyan, et al., 2004:Use of probability distribution functions for discriminating between cloud and aerosol in Lidar backscatter data, Journal of Geophysical Research: Atmospheres (1984-2012), D15,109.

[4] Sassen K, Zhao H., 1993: Super cooled liquid water clouds in Utah winter mountain storms: Cloud-seeding implications of a remote-sensing dataset, Journal of Applied Meteorology, 32(9), 1548- 1558

[5] Freudenthaler V, Esselborn M, Wiegner M, et al., 2009: Depolarization ratio profiling at several wavelengths in pure Saharan dust during SAMUM 2006, Tellus B, 61(1), 165-179.

[6] Dai G, Wu S, Song X, et al., 2014: Research in depolarization of particles in Tibetan Plateau and coastal area by lidar, SPIE Asia Pacific Remote Sensing. International Society for Optics and Photonics, 92620I-92620I-12.

[7] Fernald, Frederick G., 1984: Analysis of atmospheric Lidar observations: some comments. Applied optics, 23.5, 652-653

[8] Althausen D, Engelmann R, Baars H, et al., 2009: Portable Raman lidar PollyXT for automated profiling of aerosol backscatter, extinction, and depolarization, Journal of Atmospheric and Oceanic Technology, 26(11): 2366-2378.

[9] Sassen K, Zhao H., 1993: Super cooled liquid water clouds in Utah winter mountain storms: Cloud-seeding implications of a remote-sensing dataset. Journal of Applied Meteorology, 32(9) 1548-1558.

[10] Toon, O., Tabazadeh, A., Browell, E. and Jordan, J., 2000: Analysis of Lidar observations of Arctic polar stratospheric clouds during January 1989, J. Geophys. Res. 105, 20 589-20 615 